\title{
Los retos de las publicaciones científicas españolas en el desarrollo de la Europa del conocimiento
}

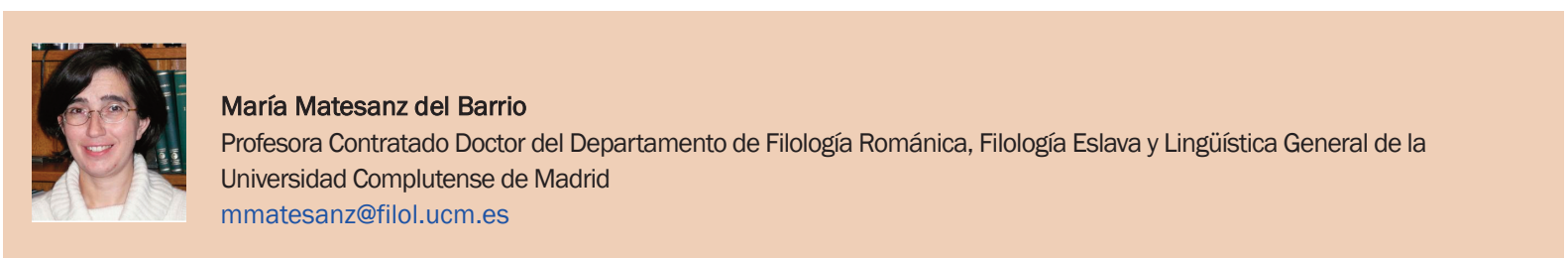

|Fecha presentación: 25/05/2010 | Aceptación: 05/10/2010 |Publicación: 17/12/2010

\begin{abstract}
Resumen
Las publicaciones científicas se están viendo afectadas por las transformaciones que se han producido en la última década a partir de la creación del EEES y EEI. Uno de los objetivos prioritarios de la Unión Europea es conseguir una Europa del conocimiento vertebrada por la actividad universitaria. En este contexto, las publicaciones científicas juegan un papel destacado como exponente de la producción científica y medio autorizado de difusión. Sin embargo, la actividad científica europea medida a través de las publicaciones especializadas no alcanza los niveles en los que se encuentran sus competidores más directos, Estados Unidos y Japón (principalmente en biomedicina y ciencias de los materiales, respectivamente).
\end{abstract}

Palabras clave: revistas científicas, EEES, EEI, criterios de calidad, investigación e innovación

\section{Resum}

Les publicacions científiques s'estan veient afectades per les transformacions que s'han produit en l'última dècada a partir de la creació del EEES y EEI. Un dels objectius prioritaris de la Unió Europea és aconseguir una Europa del coneixement vertebrada per l'activitat universitària. En aquest context, les publicacions científiques juguen un paper destacat com exponent de la producció científica i mitjà autoritzat de difusió. No obstant, l'activitat científica europea mesurada mitjançant les publicacions especialitzades no arriba als nivells en què es troben els seus competidors més directes, Estats Units i Japó (principalment en biomedicina i ciències de les materials, respectivament).

Paraules clau: revistes científiques, EEES, EEI, criteris de qualitat, investigació i innovació

\begin{abstract}
The scientific publications are being affected by the transformations that have taken place in the last decade from the creation of the EHEA and ERA. One of the priority aims of the European Union is to obtain a Europe of the knowledge, with the backbone provided by the activity of the university. In this context, the scientific publications play a key role as prime example of the scientific production and authorised way of diffusion. Nevertheless, the European scientific activity measured by the specialised publications does not reach the levels of its most direct competitors, The United States and Japan (mainly in Biomedicine and Materials Science, respectively).
\end{abstract}




\section{Introducción}

Las revistas científicas españolas se encuentran en un momento de indudables y necesarios cambios con el fin de ser competitivas en el contexto europeo. El análisis que se realiza en este artículo no pretende ser exhaustivo pero sí lo suficientemente ilustrativo como para conocer la realidad actual de estas publicaciones. En algunos aspectos, la descripción puede resultar alarmante para muchas de nuestras publicaciones porque, sin querer ser en absoluto catastrofista, parece más que probable que desaparezca un buen número de ellas en un plazo no demasiado largo, si no de forma real sí en el plano del prestigio científico, lo que hará de ellas revistas no competitivas carentes de visibilidad. No comparto la opinión de quienes piensan que para las revistas de algunas ramas de conocimiento, como las que recogen la investigación en Ciencias Experimentales, la situación es posiblemente irreversible, y algo más favorable en Humanidades (Guillamón 2006: 5). Algunos datos que aporto, ciertamente, apoyan esta tesis, pero también es verdad que la situación de cambio, común a todas las revistas europeas, puede permitirnos, hasta cierto punto, reconducir la actual tendencia 0 , al menos tener más presencia científica a través de nuestras publicaciones, que deben aspirar a la transnacionalidad. Renovación, reorganización e innovación, en el marco de las propuestas europeas sobre publicaciones científicas, deben ser criterios que orienten el futuro de las publicaciones científicas españolas.

La adopción de los principios de la Declaración de Bolonia (1999) es ya una realidad en los nuevos planes de estudio de grado y máster que se han empezado a implantar el curso 2009-2010 en las universidades ${ }^{1}$. Si de una manera más o menos directa los profesores de las universidades europeas conocemos, con cierto detalle, todo el proceso de adaptación de los estudios al Espacio Europeo de Educación Superior (EEES), no ocurre lo mismo con el desarrollo del Espacio Europeo de Investigación (EEI). La propuesta de creación de este Espacio se produjo poco después (Estrategia de Lisboa, 2000) ${ }^{2}$, pero su evolución no ha sido tan rápida ni ha dado resultados tan concretos y perceptibles para la comunidad científica como ha sucedido con el EEES. Esta diferencia de desarrollo se ha hecho patente, en general, en una implicación menor de los profesores universitarios en el seguimiento de la evolución e implantación de las políticas conducentes a la consolidación del EEI ${ }^{3}$, a pesar de que afecta de forma directa a su faceta investigadora.

La década que ha transcurrido desde la Declaración de Bolonia deja todavía ámbitos en los que quedan caminos por explorar ${ }^{4}$ para que esa Europa del conocimiento ${ }^{5}$ sea una realidad que vertebre toda la actividad universitaria. El análisis de la Comisión sobre las universidades reconoció en estas instituciones el centro neurálgico de la investigación, educación e innovación que posibilitan la construcción y desarrollo de la economía y sociedad del conocimiento (CCE 2002: 5). Además, las universidades contribuyen a la consecución de otros objetivos estrechamente relacionados: empleo, cohesión social y mejora del nivel educativo. El papel clave que la Unión Europea ha otorgado a la Universidad hace que ante las universidades se hayan abierto nuevos desafíos, lo que ha supuesto necesarias adaptaciones. El momento en el que nos encontramos al realizar esta reflexión nos permite una mirada casi equidistante a dos décadas de proyectos renovadores en la misión, organización y evolución de las universidades: desde la Declaración de Bolonia hasta 2010 y desde esta fecha hasta 2020. La primera década del siglo XXI, que ha culminado con la conocida convergencia del proceso de Bolonia, ha estado marcada por cambios profundos en seis grandes líneas de acción: (i) aumento de la demanda de formación superior; (ii) internacionalización de la educación y la investigación; (iii) cooperación estrecha y eficaz entre la universidad y la industria; (iv) multiplicación de los lugares de producción de conocimientos; (v) reorganización de los conocimientos; (vi) formación a lo largo de la vida. Los próximos diez años estarán marcados por tres ejes orientados a conseguir que el conocimiento sea el motor del crecimiento sostenible: (i) investigación, (ii) innovación y (iii) creatividad y con unas iniciativas claras de actuación muy definidas, como veremos (CCE 2010).

En el marco que presentamos, el papel que juegan las publicaciones científicas es fundamental como exponente y difusor del conocimiento científico, como plataforma de cohesión de esfuerzos investigadores y como estímulo para el crecimiento de la actividad investigadora. Pero no todas las revistas están en situación de afrontar los nuevos retos que se han marcado desde el EEES y el EEI. Es fácil constatar una falta de posturas comunes de investigadores y docentes ante la necesaria renovación de las revistas, y se impone la reflexión y discusión de bases comunes que permitan reconocer las publicaciones españolas como elementos integrantes de la Europa del conocimiento.

\section{Consideraciones sobre las revistas científicas españolas en Europa}

La producción científica en Europa está a la cabeza de la producción mundial y sigue una línea ascendente. En 2004, un $41,3 \%$ de la totalidad de publicaciones se producían en Europa, frente a un 31,4 \% en Estados Unidos, según los datos aportados por la Comisión Europea para esa fecha ${ }^{6}$.

\footnotetext{
${ }^{1}$ La implantación de nuevos estudios en el marco del EEES no agota las posibilidades del Espacio de Educación Superior, como lo demuestra la reciente Conferencia de Budapest-Viena, 2010 y la planificación de la próxima reunión de ministros en Bucarest en abril de 2012.

${ }^{2}$ La Unión Europea adoptó la Estrategia de Lisboa con la perspectiva de convertirse en la economía del conocimiento más competitiva y más dinámica del mundo. El antecedente de esta declaración lo constituye el Comunicado de la Comisión Hacia un espacio europeo de investigación (CCE 2000a) y los planteamientos generales se recogen en el documento Realización del "Espacio europeo de la investigación" (CCE 2000b).

${ }^{3}$ De los documentos europeos oficiales que han dado coherencia y continuidad a este espacio de educación e investigación consideramos de especial relevancia los siguientes: Declaración de Bolonia, 1999; Estrategia de Lisboa, 2000; Comunicado de Berlín, 2003; Estrategia de Lisboa renovada, 2005; Libro Verde (CCE 2007b y CCE 2007c); Conclusiones del Consejo europeo de 13 y 14 de marzo de 2008 (“quinta libertad"); Proceso de Liubliana (2008); Un Plan Europeo de Recuperación Económica (CCE 2008); Visión 2020 para el Espacio Europeo de Investigación, 2008; Consulta sobre la futura estrategia "UE 2020, 2009 (CCE 2009)".

${ }^{4}$ En el último año de la década que consideramos, 2009, los estudios y análisis de la comunidad se han centrado, entre otros asuntos, en las políticas que impulsan I+D y el impacto de la colaboración en Europa entre ciencia y tecnología (Kroll y Stahlecker 2009; Kroll, Zenker y Schubert 2009; Peter y Frietsch 2009).

${ }^{5}$ Esta es la denominación que se da en el Comunicado de Berlín (2003: 5) al Espacio Europeo resultante de la confluencia del EEES y del EEl.

${ }^{6}$ Se ofrecen también datos sobre el volumen total de producción científica: “The number of scientific periodicals published throughout the world exceeds
} 20 000. Every year, 1.5 million scientific articles are published worldwide". (IP/04/747: p. 1). 
Datos más recientes, ofrecidos por la Comisión en 2007 presentan una situación más favorable, y de los alrededor de 2000 editores de revistas científicas (que producen aproximadamente 1,4 millones de artículos al año), unos 780 están situados en la Unión Europea (UE), correspondiéndoles el 49 \% de la producción total de revistas (CCE 2007a: 3). Además, en 2006 el 37,6 \% de la producción mundial de los artículos científicos sometidos a revisión por pares estaba firmado por, al menos, un autor de la UE frente al 31,5 de Estados Unidos (CCE 2008: 61). Sin embargo, estas cifras no tienen correlato con el número de referencias de sus publicaciones, puesto que, globalmente, es menor. Hay que tener en cuenta, además, que si consideramos las cifras por países, algunos países europeos tienen una presencia completamente insignificante en números absolutos. Las cifras muestran la baja tasa de representatividad de las publicaciones europeas en relación con la totalidad de la producción mundial, siendo España, además, uno de los países que está por debajo de la media europea ${ }^{7}$. De acuerdo con los datos del Plan Nacional 2008-2011 (2007: 24), en España en 2006 el número de trabajos realizado en instituciones españolas y publicados en revistas del Science Citation Index (SCI), en las que al menos un autor pertenecía a la institución, fue de 36 840, el 3,1\% de la producción mundial.

Como es sobradamente conocido, en la evaluación de las publicaciones científicas ${ }^{8}$ se manejan criterios de calidad, fijados con parámetros bastante estandarizados. Aunque con cierta variación en el número de criterios, su ordenación y priorización, los indicadores de calidad se pueden organizar en cinco grupos: (i) criterios de calidad editorial, es decir, criterios que afectan a todo los aspectos ligados al paratexto; (ii) calidad de los contenidos; (iii) capacidad de atracción -interés que suscita la publicación entre los autores como objetivo de publicación-; (iv) calidad de difusión, visibilidad y accesibilidad, en particular, presencia en bases de datos; y (v) calidad de repercusión, centrada prioritariamente en los índice de impacto. La presencia de las revistas en bases de datos reconocidas adquiere una singular relevancia, dada la importancia que han adquirido estos índices. Su uso trasciende el criterio de difusión de una publicación e incide también en el impacto de los artículos publicados, del autor de esos artículos, de las instituciones y del país en los que se produce ${ }^{9}$.

La explicación de la escasa representatividad de las revistas científicas españolas en los índices ${ }^{10}$, en particular de las revistas de Humanidades, responde a causas que han sido bien identificadas: reticencias en el uso del inglés, baja profesionalización de los equipos editoriales, falta de regularidad en la publicación, distribución limitada y escaso o insuficiente control científico de los contenidos (Pérez Sedeño 2005: 4). De las razones señaladas, creo que merece una particular atención la cuestión del uso del inglés, por los cambios que ha producido en las publicaciones nacionales de países de habla no inglesa y por el debate que suscita.

No cabe duda de que la lengua es un factor muy importante para la difusión de los trabajos científicos y el inglés es, incontestablemente, la lengua de comunicación científica, al igual que ocurre en otros sectores. No obstante, conviene hacer la reflexión de que publicar en inglés supone, para muchos de los investigadores que no son anglófonos, una dificultad añadida que no debe ser desdeñada, puesto que puede ser un elemento que influya negativamente en la calidad de los artículos, ya que la legibilidad es un factor que se tiene en cuenta al valorar un trabajo, y no siempre los artículos están redactados en un nivel de corrección lingüística de lengua primera (L1). Aunque no dispongo de datos precisos sobre la competencia lingüística en inglés de los investigadores españoles, no creo que los datos difieran mucho de los recogidos en Francia sobre esta cuestión ${ }^{11}$. El último informe elaborado para el Parlamento por la Délégation générale à la langue française et aux langues de France ofrece datos concretos sobre el uso del inglés entre científicos, reconocido por un 92\% de los investigadores como la lengua internacional más utilizada, pero con un uso real en Francia bastante inferior (Rapport 2009: 58-62). En la síntesis de este informe, el inglés es reconocido por los entrevistados (directores de laboratorios, investigadores y profesores-investigadores) como lengua internacional de máximo uso en ciencias exactas y naturales, sobrepasando el $70 \%$ en un uso exclusivo, frente al $34 \%$ en Ciencias Sociales y Humanidades (Rapport. Synthèse 2009: 6) ${ }^{12}$.

\footnotetext{
${ }^{7}$ No obstante, en el último informe ERAWATCH sobre las políticas de I+D en el EEl, sin dar cifras precisas, se considera que el nivel de producción de conocimiento ha aumentado en el sector académico y los resultados de la actividad investigadora, en términos de publicación, tienen un nivel satisfactorio, no así la producción de patentes (Heijs 2009: 16).

${ }^{8}$ La evaluación de las publicaciones científicas se realiza, fundamentalmente, desde tres ámbitos diferentes: (i) instituciones públicas encargadas de valorar la actividad investigadora del personal docente e investigadores de centros públicos y privados; (ii) bases de datos bibliográficas cuyo objetivo es la difusión de información científica de alto nivel; (iii) bibliotecas y centros de documentación, por la necesidad interna de disponer de información relevante para aplicar políticas de adquisición y cancelación adecuadas al centro en el que están ubicadas, y por la finalidad misma de las bibliotecas, ofrecer a los usuarios información necesaria para su trabajo. Los propios autores de los artículos científicos manejan los criterios ya establecidos. Para detalles sobre el análisis de los criterios de evaluación puede consultar el extenso estudio de Delgado López-Cózar, Ruiz-Pérez y Jiménez-Contreras (2006).

9 "The JIF [ISI Journal Impact Factor] is now commonly used to measure the impact of journals, and by extension the impact of the articles they have published, and by even further extension the authors of these articles, their departments, their universities and even entire countries" (Bollen, J. et al., 2009: 1).

${ }^{10}$ Son solamente 90 las revistas registradas en 2009 entre JCR Science Edition (59) y Social Science Edition (31), aunque ha habido un notable aumento respecto de 2008, únicamente con 53 revistas (Roldán López 2010). No entramos en este estudio en una cuestión que tiene casi tanta importancia como la entrada en los registros del JCR, el cuartil en el que se sitúa una revista en su campo, ya que muchos investigadores solamente consideran de interés para publicar las revistas que se sitúan en el primer cuartil.

${ }^{11}$ Los datos más recientes sobre el uso de las lenguas en la Unión Europea (CCE 2005b; Eurobarómetro 64.3, 2006) resultan bastante ilustrativos a este respecto. El $56 \%$ de los ciudadanos europeos afirma poder mantener una conversación en una lengua distinta de la materna, y el $28 \%$ en dos lenguas, aunque el $44 \%$ no conoce más que su lengua materna. El caso de España, en cuanto a la posibilidad de mantener una conversación en una lengua diferente de la materna, está por debajo de la media europea y por debajo de países como Francia (51\%), Polonia (57 \%) o Alemania (67 \%). Aunque el inglés es la lengua extrajera en la que un mayor número de ciudadanos es capaz de mantener una conversación (38\%), en España solamente se alcanza el 27\% (Eurobarómetro 64.3, 2006: 8-13). Evidentemente, los profesores e investigadores constituyen una élite en cuanto a su formación, pero conviene señalar que parece claro que muchos de ellos tienen que realizar un esfuerzo suplementario para estar en un nivel superior al de la media de otros ciudadanos europeos cuya formación en lenguas es superior.

12 Las encuestas presentadas en el Rapport (2009) sobre la competencia en seis lenguas naturales muestran el uso del inglés como prioritario, pero con una diferencia grande de uso en las distintas disciplinas, que oscila entre el $82 \%$ en física y un $52 \%$ en matemáticas, debido a la tradición de estos estudios en Francia. El dominio del inglés entre los investigadores también es desigual. El 3\% de los investigadores tienen dificultades para leer en inglés y 18\% en comprensión oral. El $29 \%$ de los investigadores experimentan dificultades en la expresión oral y 29\% igualmente en expresión escrita. Solamente el 37\% de los investigadores no tiene dificultad en el manejo de lenguas, mientras que el $42 \%$ encuentran dificultades en inglés. Por último, el $55 \%$ de los investigadores dicen que no han intentado mejorar sus conocimientos y el $32 \%$ dicen haberlo hecho, pero solamente para el inglés.
} 
En el lado opuesto, el monolingüismo de parte de los investigadores ingleses representa también una limitación para el desarrollo de de su actividad investigadora. Merece la pena hacer referencia a la llamada de atención que ha hecho a este respecto la British Academy a raíz del estudio encargado para conocer la situación del aprendizaje de lenguas extranjeras en el Reino Unido en todos los niveles (British Academy 2009). Por lo que se refiere a los investigadores en Ciencias Sociales y Humanidades, el informe revela la posición de desventaja en la que se encuentran al no conocer otras lenguas. Esta situación les lleva a limitar la elección de temas para sus trabajos de investigación y a tener dificultades para sus colaboraciones en proyectos internacionales, lo que perjudica los propios intereses británicos (Levitt et al., 2009: xiii-xvii).

$\mathrm{Al}$ margen de las consideraciones precedentes sobre la competencia lingüística de los científicos, es indudable que la introducción del inglés constituye uno de los cambios en las publicaciones experimentados en el panorama mundial en países que no son de habla inglesa. Sin embargo, este cambio no es por sí mismo motivo suficiente para que una revista entre a formar parte de las bases de datos de publicaciones científicas. Una mirada rápida a algunos de los campos de ciencias o ciencias sociales del Journal Citation Reports (JCR) de $2009^{13}$ nos revela varios datos de interés para lo que tratamos. Uno de ellos, que salta rápidamente a la vista, es el uso predominante del inglés en las publicaciones, pero hay otros datos más relevantes que merecen un análisis más detallado.

De los numerosos campos en los que están organizados los índices del JCR hemos elegido para nuestro análisis el campo Biochemistry and Molecular Biology, por varias razones. Por una parte, porque es el campo que tiene el mayor número de registro de revistas (283), aunque otros como, por ejemplo, Engineering Electrical \& Electronics (245) o Neurosciences (230), constan también de un número amplio. Por otra, porque tiene una alta representación de países del mundo al ser un tipo de investigación a la que, en general, todos los países dedican muchos recursos. La consulta del campo Biochemistry and Molecular Biology (JCR 2009) aporta los siguientes datos sobre un total de 283 publicaciones registradas: (i) de los 23 países con publicaciones indizadas en este campo, solamente en seis de ellos el inglés es la lengua oficial o cooficial con otra u otras lenguas ${ }^{14}$; (ii) 186 revistas proceden de países de lengua inglesa, principalmente de EE.UU. y Reino Unido ${ }^{15}$, por este orden; (iii) el número de revistas en este campo de los países pertenecientes a la Unión Europea, exceptuando el Reino Unido, Holanda y Alemania ${ }^{16}$, no llega a cinco y de España solamente hay una ${ }^{17}$; (iv) en el campo lengua, solamente hay 16 publicaciones en las que se ha anotado su carácter multilingüe (que, en general, significa bilingüe, siendo el inglés una de las lenguas); y (vi) solamente una de ellas es monolingüe en una lengua diferente del inglés, en chino ${ }^{18}$. Los datos no son extra-

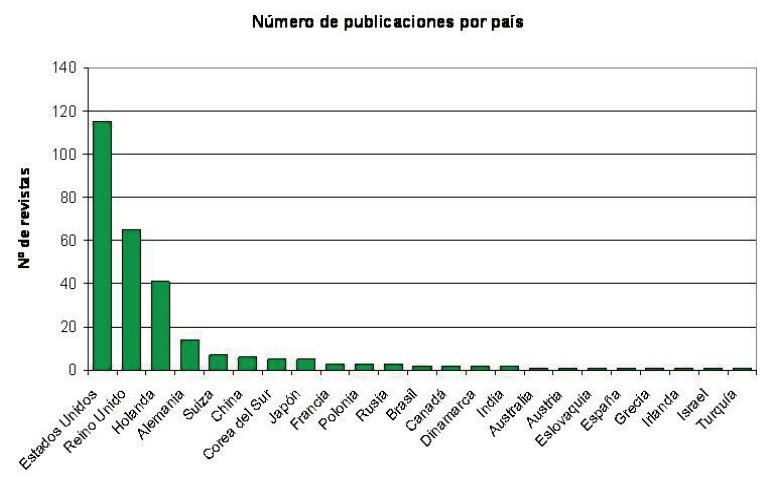

Figura 1. Datos del campo Biochemistry and Molecular Biology (JCR 2009).

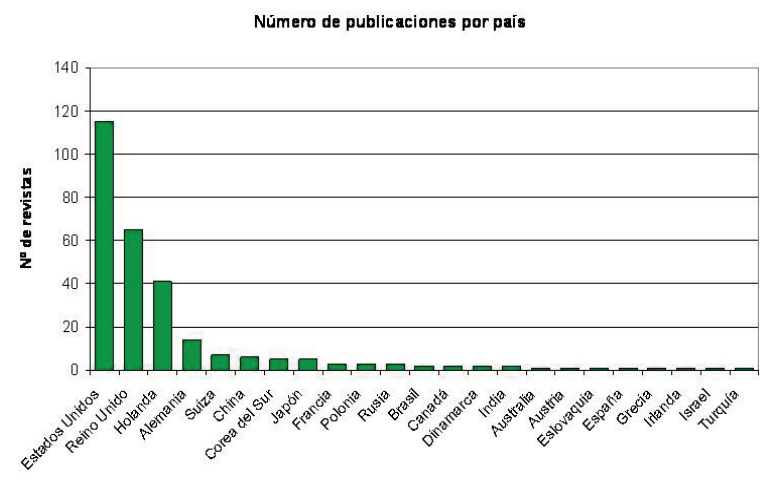

Figura 2. Datos obtenidos de la combinación del campo Biochemistry and Molecular Biology (JCR 2009) y censo de población mundial del IDB ${ }^{19}$

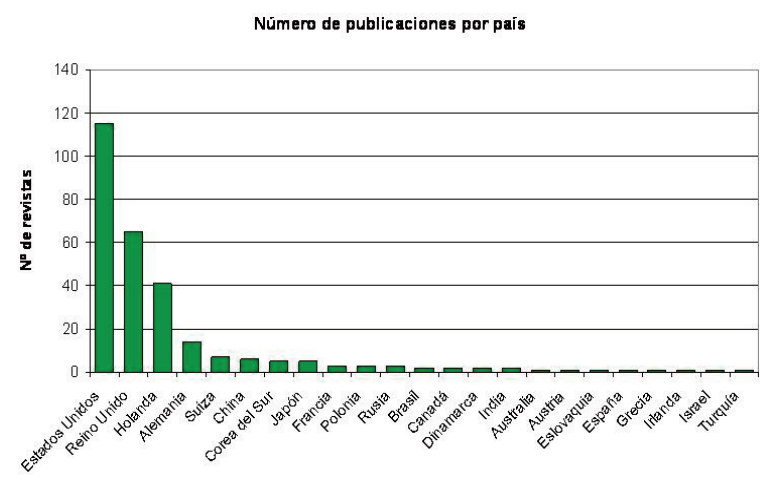

Figura 3. Datos obtenidos de la combinación del campo Biochemistry and Molecular Biology (JCR 2009) y del PIB por países ${ }^{20}$.

\footnotetext{
${ }_{13}$ Últimos datos publicados en el momento de escribir este artículo.

${ }^{14}$ Los países en los que se han editado una o más revistas incluidas en este índice son los siguientes: Alemania, Australia, Austria, Brasil, Canadá , China, Corea del Sur, Dinamarca, Eslovaquia, España, Estados, Unidos, Francia, Grecia, India, Irlanda, Israel, Japón, Países Bajos, Polonia, Reino Unido, Rusia, Suiza, Turquía.

${ }^{15}$ Además de Irlanda y países en los que el inglés coexiste con otra u otras lenguas. La producción de EE.UU. en este campo es de 115 revistas y en el Reino Unido se editan 65.

${ }_{16}$ Para el campo Biochemistry and Molecular Biology Holanda y Alemania cuentan con 41 y 14 publicaciones, respectivamente.

${ }^{17}$ La revista registrada es Journal of Physiology and Biochemistry, en el puesto número $235(1,172)$ y es de carácter multilingüe (bilingüe).

${ }_{18} \mathrm{De}$ las seis revistas de China en este apartado solamente una de ellas, Progress in Biochemistry and Biophysics, se publica en chino, pero su índice de impacto es el segundo más bajo $(0,211)$, en el puesto 282. En el índice de 2009 se ha incluido la revista china Journal of Huazhong University of Science and Technology [Medical Sciences], publicada en inglés y con algunos artículos en alemán.
} 
polables al conjunto de la producción científica publicada en revistas, pero son, ciertamente, reveladores. De particular interés son las cifras comparativas que podemos extraer cuando valoramos las publicaciones en función del número de habitantes de cada uno de los países que figura en el campo seleccionado y con el producto interior bruto (PIB) de ellos.

Las Figuras 1, 2 y 3 muestran la periférica posición de las revistas españolas en el campo Biochemistry and Molecular Biology, sobre todo en relación con el PIB, como hemos señaldo. Menos de la mitad de las revistas del campo Biochemistry and Molecular Biology registradas en el JCR 2009 se publican en la UE. En realidad, solamente once países de los 27 tienen revistas indizadas y, además, existe una clara desigualdad entre la producción de estos once países. Con relación al año 2008, la situación respecto de Estados Unidos y Japón apenas ha variado ya que de las nueve revistas que por primera vez entran en Biochemistry and Molecular Biology dos están publicadas en EE.UU. y cuatro en la UE, aunque la diferencia real es de dos porque han salido del índice un título de Rumanía y otro del Reino Unido.

En una ojeada rápida a los índices del JCR de distintas áreas vemos que, en su conjunto, el número de publicaciones en español registradas es bajísimo y, además, no siempre son publicaciones realizadas en España. Así, por ejemplo, para el campo Linguistics hasta el índice de 2009, en el que ha entrado a formar parte de él la Revista Española de Lingüística Aplicada, solamente había una publicación en lengua española, Revista Signos, publicada en Chile. Las revistas consideradas multilingües y en las que una de las lenguas es el español no se producen necesariamente en España, como en el caso anterior o, por citar otro ejemplo, el de Revista de Bilología Tropical, perteneciente al campo Biology, que se edita en Costa Rica y cuya estructura fija se organiza sobre dos elementos lingüísticos, (i) resumen en inglés y español y (ii) texto de los artículos en una de las dos lenguas. Los datos para otros países europeos de nuestro entorno, como Francia o Italia, no son mucho mejores y las publicaciones en francés ${ }^{21}$ o italiano apenas tienen representación.

El caso de Geologica Acta ${ }^{22}$ es un claro ejemplo de la importancia que tiene publicar en inglés, pero muestra, a su vez, que éste no es un requisito imprescindible para entrar en las bases de datos tenidas en cuenta en los índices de impacto $^{23}$. Esta revista española se publicó en el período 19662002 bajo el nombre de Acta Geologica Hispanica, gracias a la colaboración del Institut de Ciències de la Terra Jaume Almera (CSIC) y la Facultat de Geología de la Universidad de Barcelona. A partir de marzo de 2003 la revista pasa a publicarse íntegramente en inglés con el nuevo título Geografica Acta. Este cambio se produce tras un breve período (1998-2002) en el que se habían incrementado considerablemente las contribuciones en inglés: un $43 \%$ en español frente a un $49 \%$ en inglés; el resto corresponde a francés y catalán $^{24}$. Solo un análisis simplista de este hecho establecería una relación causa efecto entre su publicación íntegra en inglés y su actual índice de impacto en el JCR (2.245), en el que ocupa la posición 6 de 42 para la categoría Geology. El paso definitivo de español a inglés se produjo junto con otras transformaciones que afectan, de modo general, a la calidad del contenido, de la propia edición y a la eficacia en su distribución por medio del libre acceso en internet ${ }^{25}$. Estos cambios ya se venían gestando en la etapa anterior de la revista, como hemos indicado, especialmente a partir de los años noventa ${ }^{26}$.

La realidad del bajo número de publicaciones españolas en las bases de datos no se debe tanto a la cuestión lingüística, aunque éste sea en ocasiones el argumento esgrimido, como a la confluencia de distintos parámetros ligados a la calidad científica y formal. El análisis de los datos aportados por las bases de datos de indexación de revistas científicas lleva a una primera conclusión evidente, al menos aparentemente: todas aquellas revistas que no figuran en ellas es porque no cumplen los estándares de calidad fijados para ser registradas en bases de datos de prestigio. La ausencia de índice de impacto de una publicación tiene consecuencias directas, relacionadas con la anterior y muy negativas para los investigadores, en particular, en algunas ramas de conocimiento. El uso de estas bases de datos, por parte de las instituciones públicas que tienen que valorar la actividad de los investigadores ${ }^{27}$ determina, grosso modo, que aquéllas que no estén indexadas o que tengan bajo índice de impacto no son tenidas en cuenta. La explicación es clara, la estandarización y fijación de criterios de calidad para las publicaciones y reuniones científicas está, actualmente, muy definida

\footnotetext{
19 International Data Base (IDB), U.S. Census Bureau, Population Division (Fecha de consulta: julio 2010).

${ }^{20}$ Datos oficiales del Fondo Monetario Internacional para 2008 (http://imf.org/external/pubs/ft/weo/2009/02/weodata/index.aspx).

${ }^{21}$ La preocupación por publicar y cómo hacer para que un manuscrito sea aceptado genera también en otras lenguas bibliografía, como lo muestra la reciente obra de Cossette (2009).

${ }^{22}$ Todos los datos sobre esta publicación con los que ha trabajado los he obtenido, principalmente de la página web http://www.geologica-acta.com/HomeAC.do ${ }^{23}$ De las 38 revistas españolas incluidas por vez primera en el JCR 2009 (Roldán López 2010), nos hemos encontrado con que una minoría aquellas está exclusivamente en inglés. La mayoría son multilingües, en general en bilingües español e inglés, con predominio, en muchos casos, del español. Además, se han incluido algunos títulos que se publican exclusivamente en español. Merece reseñarse Trabajos de Prehistoria, solamente en español, que ha entrado en el segundo cuartil, lo que no es habitual. También ha entrado en el segundo cuartil Revista Matemática Complutense, que actualmente se edita únicamente en inglés.

${ }^{24}$ En el período precedente considerado por la propia publicación (1994-1997) la situación es diametralmente opuesta, unos 70 artículos en español frente a algo más de 10 artículos en inglés. Es particularmente interesante contrastar estos datos con los que presentan sobre la nacionalidad del primer autor, mayoritariamente española antes del paso a la nueva publicación. Entre 1988 y 2002, el 62\% de los artículos publicados tenían como primer autor a un español, lo que revela, claramente, la apuesta de los autores españoles por escribir en inglés como medio más eficaz de llegar a un número mayor de lectores especialistas en su ámbito.

${ }^{25}$ Son varios los aspectos que destacan como claves para la nueva revista, entre los que señalamos: (i) en el contenido: desarrollos conceptuales relevantes en cualquier área de Ciencias de la Tierra; monográficos sobre resultados de investigación de uno o más grupos; resultados parciales de trabajos en curso; contribuciones a congresos, etc.; (ii) nuevo formato, publicación íntegra en inglés, ampliación de miembros del comité científico, aumento de revisores externos; (iii) publicación rápida en libre acceso permanente.

${ }^{26}$ En la página web de la revista se puede acceder a un breve resumen histórico de la publicación en el que se muestra su evolución. A partir de los años noventa, la revista se consolida dentro de su área, aumenta el número de artículos con impacto, las contribuciones de equipos internacionales de investigación y se generaliza la inclusión de un resumen amplio para artículos escritos en español o portugués.

${ }^{27}$ No entramos en la interesante cuestión, señalada recientemente por D. Fanelli, del conflicto que supone para los investigadores la presión de publicar para poder ser valorado académicamente y la calidad de los resultados de sus investigaciones (Fanelli, 2010).
} 
Criterios de calidad de las publicaciones científicas en el EEES ${ }^{28}$

1. Indicadores para la evaluación de las revistas científicas: criterio fundamental para su evaluación à calidad

a. Prestigio del editor

b. Prestigio del equipo editorial

c. Difusión, visibilidad y accesibilidad

(i) Versión electrónica

(ii) Presencia en bases de datos

(iii) Presencia en bibliotecas del EEES, nacionales e internacionales.

(iv) Circulación: tirada, suscripciones e intercambios.

d. Tipología de los contenidos à se valora

(i) El proceso de selección de los artículos

(ii) El carácter temático de las publicaciones, identificando las calidades informativas y explicativas del texto que debe - Ser de carácter innovador

- Ser riguroso metodológicamente

- Ser de actualidad y de relevancia

- Contener resúmenes, palabras claves y traducción al inglés del sumario

e. Se ajustan a las normas ISO, AENOR, UNESCO

f. Parámetros de evaluación

(i) Regularidad: se valora la publicación sin interrupciones

(ii) El ISSN

(iii) Tipo de soporte, formato

2. Las publicaciones de las revistas del EEES debe corresponder esencialmente a revistas de nivel

3. Relación entre revistas científicas y ramas de conocimiento

Cuadro 1. Criterios de calidad de las publicaciones científicas en el EEES

y son pocos los márgenes de variación. Basta consultar cualquier base de datos o la bibliografía reciente en este ámbito de análisis para tener una idea clara y rápida de cuáles son los parámetros con los que se trabaja para establecer y medir la calidad de las publicaciones.

El propio Espacio Europeo de Educación Superior maneja, parcialmente, estos criterios para evaluar las publicaciones científicas que se producen en su seno y establece una serie de indicadores cuyo principio rector es, sin duda, la calidad de la información y científica.

La consolidación de criterios generales aceptados por la comunidad científica para medir la calidad y relevancia de la investigación hace que exista una aparente homogeneidad entre las publicaciones que cumplen los estándares de calidad. Sin embargo, esta homogeneidad exigida a las publicaciones empieza a mostrar algunos puntos cuestionables. Quizá sería más apropiado decir que lo que se plantea es considerar, además, otros criterios ligados a la difusión en la red y accesibilidad de las publicaciones científicas para valorar el número de citas que recibe una publicación (Bollen et al. 2009). La propuesta de corrección de criterios, actualmente completamente aceptados, resulta interesante y puede mejorar la consideración de revistas en su conjunto y de artículos independientes. Pero, además, para casos en los que el campo de investigación es minoritario, local o, simplemente, no tienen ese reconocimiento en las bases de datos, los criterios relacionados con la indexación en general y los índices de impacto en particular deberían ser matizados. En la rama de Humanidades este tipo de situación es relativamente frecuente. El Libro blanco de la investigación en Humanidades (LB Humanidades) se hace eco de esta situación y considera, incluso, el sistema de evaluación a través de índices de impacto de "difícil y cuestionable validez" (LB Humanidades 2006: 23), aunque, en la práctica, no se ha hecho demasiado caso a esta acertada observación. No es extraño trabajar en temas en los que no hay apenas revistas indexadas o éstas tienen un índice de impacto muy bajo, y encontrarse con la paradójica situación de que publicaciones de interés y prestigiosas para los especialistas en la materia no tienen repercusión en las bases de datos de las publicaciones científicas. La investigación en lexicografía, por ejemplo, es un caso patente de esta situación, ya que solamente cuenta con una publicación indexada en el JCR y sólo desde 2007, International Journal of Lexicography. La única revista española de esta temática indexada es Revista de Lexicografía, que figura únicamente en el REGESTA IMPERII y en el ISOC. Sin embargo, cada vez tiene más visibilidad la investigación en este campo a través de otros medios y son muchos los investigadores que trabajan en metalexicografía y publican, a la vez, diccionarios y otras obras lexicográficas.

La ausencia en las bases de datos de revistas que entre los especialistas gozan de prestigio tiene consecuencias de distinto tipo. En primer lugar, (i) se presentan ante la comunidad científica como publicaciones carentes de interés científico, lo que no siempre es cierto, $y$ publicar en ellas tiene efecto negativo sobre los currículos que son juzgados por las comisiones encargadas de valorar las trayectorias de los investigadores. La consecuencia inevitable, que ya se está produciendo, es (ii) el despoblamiento de artículos de interés y calidad en esas revistas, hasta el punto de que podría llegar a provocar la desaparición de la propia publicación, puesto que (iii) muchas de ellas empiezan a tener dificultades para tener colaboraciones. Es cada vez más frecuente encontrar en las listas de distribución especializadas llamadas al envío de contribuciones en publicaciones especializadas del área, lo que es un evidente indicador de la situación en la que se encuentran muchas revistas científicas. Desconozco el dato global, si es que se dispone de él, de la proporción de autores españoles que realizan sus investigacio- 
nes en España y que eligen como primera opción revistas extranjeras para publicar sus resultados y en qué proporción lo logran. Junto a los datos de artículos aceptados de autores españoles (o entre los que figuran autores españoles) sería muy interesante conocer, aunque dudo que esto sea posible, los datos sobre el número de artículos rechazados en revistas extranjeras con alto índice de impacto y que después son publicados en revistas españolas, indexadas también, pero con menor peso.

La reacción a la situación generada se ha producido, desde mi punto de vista, con un cierto retraso, en el sentido de que muchas publicaciones consolidadas en un área no se han preocupado hasta hace poco de aplicar a sus publicaciones los controles de calidad necesarios para ser indexadas. Posiblemente, su propio estatus de revista consolidada dentro de una determinada área haya contribuido a esta despreocupación. Es sorprendente que en España publicaciones con más de medio siglo de existencia y prestigio en su área empiecen ahora a ser recogidas en bases de datos extranjeras. Algunas de las más conocidas revistas del CSIC están en esta situación, y en la página web de sus publicaciones encontramos noticia de estos cambios. Su presencia en SCOPUS, precisamente, para muchas de ellas se ha producido recientemente, a pesar de ser un referente en su área, como es el caso, por ejemplo, de Revista de Filología Española, en el índice desde 2008 o de Revista de Literatura, presente desde 2009.

A la vista de la situación en la que nos encontramos, la subsistencia de muchas de las revistas científicas que actualmente se producen en España pasa por adaptarse a nuevas formas de entender la generación, gestión y comunicación del conocimiento en Europa.

\section{Innovación en las publicaciones científicas}

La Declaración de Bolonia y la Estrategia de Lisboa, a las que nos hemos referido al inicio de este artículo, son los pilares que han servido y sirven de base para desarrollar principios comunes a todos los países comunitarios, en docencia e investigación, que permitan la identificación de sus miembros como pertenecientes a ese Espacio. De esta primera Declaración, más de principios que resolutiva, se desprenden elementos vertebradores que se han ido desarrollando en los años siguientes. El primero de estos criterios comunes de desarrollo es la calidad, ligada a la competitividad en las distintas áreas. Este objetivo de excelencia en los resultados científicos va a estar siempre presente en los documentos en los que se aborda la construcción del EEES y del EEI, especialmente en los últimos años. Si en la Declaración de Bolonia las reflexiones se centran, fundamentalmente, en la Educación Superior, en la Estrategia de Lisboa se propone la creación de una zona europea de investigación e innovación. En realidad, esta propuesta materializa las recomendaciones de la Comisión recogidas en el Comunicado Hacia un espacio europeo de investigación (CCE 2000a). En él se concede a la investigación un papel central en la aplicación de las políticas públicas y ocupa un lugar preponderante en el proceso de decisión política. El análisis de las actividades de investigación y desarrollo tecnológico reveló un panorama preocupante frente a otras potencias, como Japón o Estados Unidos. El Consejo de Europa consideró que la situación únicamente podía ser reconducida si se coordinaba una acción conjunta y, de este modo, se podría lograr la transición que se había emprendido a nivel mundial hacia la economía del conocimiento. Las reflexiones que se hacen en este y otros documentos iniciales responden a planteamientos generales y no se llega en ninguno de ellos a abordar cuestiones tan concretas como la política de publicaciones científicas, para lo que habrá que esperar unos años. Sin embargo, creo que merece la pena destacar algunas líneas generales de acción porque tienen aplicabilidad y vigencia para las publicaciones científicas. En los documentos manejados hay varios puntos que deben ser considerados y analizados en las publicaciones, si se pretende seguir las líneas definidas por el EEES y el EEI: (i) calidad de la investigación hacia la excelencia científica; (ii) fragmentación de los esfuerzos; (iii) incremento del atractivo de Europa para los investigadores del resto del mundo; (iv) necesidad de afianzar y asegurar los lazos entre el EEES y el EEI; (v) necesidad de aumentar la competitividad; (vi) difusión rápida y amplia de los resultados de la investigación para acelerar la innovación; (vii) preservación de publicaciones y datos de la investigación ${ }^{29}$; (viii) buenas prácticas en relación con los nuevos modelos de acceso a la información científica y de difusión de la misma; y (ix) transición del mundo impreso al mundo digital.

Estos principios se encuentran con mayor o menor incidencia en las tres fases que hemos considerado para la década: fase 1 (1999-2003); fase 2 (2004-2007); fase 3 (2005-2009). La primera fase determina el establecimiento de criterios básicos que se mantendrán y desarrollarán en los diez años de construcción de la Europa del conocimiento. En estos años se concede un papel preponderante a la investigación como factor clave en el logro de una economía del conocimiento. El análisis de las actividades de investigación y desarrollo tecnológico había revelado un panorama preocupante para Europa frente a potencias como Japón y EE.UU. El inicio de las reflexiones sobre la importancia y necesidad de impulsar la producción científica parte de un texto que ha marcado el verdadero cambio en la concepción de la difusión de resultados de investigación, la Declaración de Berlín (2003) 30 $^{30}$, con su propuesta de libre acceso a la información, el llamado, comúnmente, acceso abierto. La Declaración de Berlín es la culminación de tres iniciativas precedentes: (i) la Iniciativa de Budapest (2001) ${ }^{31}$; (ii) Carta ECHO (2002) $)^{32}$; y (iii) Declaración de Bethesda $(2003)^{33}$.

\footnotetext{
${ }^{29}$ En particular, este punto es sólo de la tercera fase considerada. Ver DO L 236/28.

${ }^{30}$ La Declaración ha sido firmada, desde octubre de 2003, por 281 instituciones de investigación (dato obtenido el 11/07/2010 en http://oa.mpg.de/openaccess-berlin/signatories.html) y sigue abierta a la firma. La publicación en acceso abierto permite acceder gratuitamente a aportaciones científicas, que pueden ser utilizadas siempre que se reconozca adecuadamente la autoría. Además, debe depositarse una versión completa del trabajo y todos sus materiales complementarios por lo menos en un repositorio en línea.

${ }^{31}$ La Iniciativa de Budapest para el Acceso Abierto surgió de una reunión convocada por el Instituto para una Sociedad Abierta (Open Society Institute OSI), Budapest, 1-2 de diciembre de 2001. El propósito de la reunión era impulsar el esfuerzo internacional para hacer que los artículos de investigación en todas las áreas académicas estuvieran disponibles de forma gratuita en Internet.

${ }^{32}$ La Carta ECHO (European Cultural Heritage Online), datada en Berlín, 30 de octubre de 2002, tiene por objeto definir los criterios para la adecuada explotación del potencial de los nuevos medios para la preservación de archivos académicos y educativos de exploración, así como la distribución pública del patrimonio cultural común de la humanidad a través de Internet.

${ }^{33}$ La Declaración de Bethesda es el resultado de las conclusiones de una amplia representación de los agentes implicados en la investigación biomédica (científicos, sociedades científicas, bibliotecas, editores, instituciones y agencias financiadoras) sobre cómo proceder, de forma inmediata, ante el objetivo de dotar a las publicaciones científicas de acceso abierto. Esta reunión tuvo lugar en la sede del Howard Hughes Medical Institute en Chevy Chase, Maryland (EEUU), el 11 de abril de 2003.
} 
Estas propuestas previas compartían un objetivo básico común, garantizar el acceso inmediato y gratuito a las publicaciones de investigación a través de internet. Su propuesta se dirige a la comunidad científica y agentes implicados en la política científica, financiación, gestión, difusión y conservación del conocimiento. La repercusión de esta declaración ha sido enorme y ha producido un cambio en las políticas de publicaciones científicas, auspiciado, sin duda, por la rápida implicación de la Comunidad Europea en esta iniciativa, con la adopción rápida de políticas en este sentido (EURAB 2006) aunque dista de estar generalizado el acceso abierto. Solamente se publica en acceso abierto alrededor del $15 \%$ del total de los artículos publicados en el mundo (Informe APEI 2 2008: 26).

La segunda fase, que se corresponde con los años centrales de la década, desarrolla y amplía los principios propuestos hasta ese momento, algunos de ellos derivados de la Declaración de Berlín. La contundencia del cruce de datos entre el volumen de la producción científica europea y el número de referencias de las publicaciones, muy inferior, llevó a una reflexión en profundidad sobre las posibilidades de revertir esas cifras $^{34}$. Una de las acciones emprendidas por la Comisión es el encargo, en 2004, de un estudio a fondo sobre la evolución económica y técnica de los mercados de publicación. Los objetivos prioritarios de este informe eran (i) determinar las condiciones necesarias para el funcionamiento óptimo del sector de las publicaciones científicas y (ii) evaluar en qué medida la Comisión podía contribuir a satisfacerlas. Algunas de las principales cuestiones que se abordan en él son el futuro de la revista científica impresa, los riesgos de accesibilidad para los investigadores debido al aumento del precio de las publicaciones, o el libre acceso a los resultados de la investigación y cómo conciliar los derechos de los autores y los intereses económicos de los editores. Los resultados de este extenso estudio se publicaron en enero de 2006, con el título Study on the economic and technical evolution of the scientific publication markets in Europe ${ }^{35}$. En él se tratan aspectos muy concretos de las publicaciones científicas, como son, por ejemplo, el impacto del IVA, las publicaciones electrónicas o el uso de estándares en lenguajes de marcado. En él se formula un decálogo de recomendaciones que deben ser tenidas en cuenta para garantizar el futuro de las revistas científicas (Study 2006: 87-89), entre las que destacamos la necesidad garantizar el acceso público a los resultados de la investigación financiada con fondos públicos poco tiempo después de la publicación de los mismos; el acceso continuo a las publicaciones académicas digitalizadas; el fomento e impulso de herramientas flexibles para mejorar la visibilidad del conocimiento, su accesibilidad y difusión; la creación del "ranking de calidad extendida" en las revistas científicas; o, en otro orden de cosas, el establecimiento de un nuevo modelo de negocio en la publicación.

Los distintos documentos generados por la Comunidad Europea sobre el EEI hasta $2007^{36}$ se analizan, y hasta cierto punto se sintetizan, en el Libro Verde (2007), en el que se pro- ponen una serie de objetivos prioritarios con el fin de profundizar y ampliar el EEI para contribuir plenamente a la Estrategia de Lisboa renovada: (i) buena circulación de investigadores competentes; (ii) infraestructuras de investigación de categoría mundial; (iii) instituciones de investigación excelentes; (iv) puesta en común efectiva de los conocimientos; (v) buena coordinación entre los programas y las prioridades de investigación; (vi) amplia apertura del Espacio Europeo de Investigación al mundo ${ }^{37}$.

A partir de 2007 (fase 3), las referencias a las publicaciones científicas son tangenciales, ligadas a aspectos más generales, como son la accesibilidad del conocimiento en Europa y su libre circulación, lo que se ha dado en llamar la "quinta libertad" (Quinta libertad 2008). Actualmente, todas las propuestas que se realizan sobre el EEI se enmarcan en el Proceso de Liubliana, acordado en abril de 2008 (Informal Meeting, 2008), y en cuyas primeras conclusiones de mayo de ese mismo año se pretende establecer una mejor administración del Espacio Europeo de Investigación (Proceso de Liubliana, 2008). Se destaca, especialmente, la necesidad de desarrollar para el EEI una visión a largo plazo sobre la base de los objetivos de la Estrategia de Lisboa. En el documento Visión 2020 se hace mención expresa de líneas que hay que seguir para conseguir la excelencia en la investigación y la competitividad de Europa en el contexto mundial. Para ello se incide en cuatro principios: (i) el EEI como soporte y garante del desarrollo y la competitividad de Europa; (ii) modernización de los sistemas de investigación, a la par que se que los sistemas de enseñanza e innovación (se vuelve hacia las interacciones en el triángulo del conocimiento); (iii) ayuda coordinada a los investigadores y a las instituciones de investigación comprometidas con la excelencia de la investigación; y (iv) refuerzo de las capacidades científicas y tecnológicas en toda Europa. Esta década se cierra con la consulta sobre la consulta sobre la futura estrategia UE 2020 (CCE, 2009), uno de cuyos principios es crear valor basando el crecimiento en el conocimiento: educación, innovación, creatividad.

Una cuarta fase se acaba de iniciar en el año 2010, considerado por la Comunidad Europea como punto de inflexión en la crisis económica de los últimos años, y que va a desarrollarse hasta 2020. En Estrategia Europa 2020 (CCE 2010) se reconocen cinco objetivos generales que deben transformarse en objetivos nacionales: (i) el empleo, (ii) la investigación y la innovación, (iii) el cambio climático y la energía, (iv) la educación y (v) la lucha contra la pobreza. Son tres las líneas de acción prioritarias de la Estrategia Europa 2020 para la consecución de sus objetivos que, además, se entrelazan: (i) crecimiento inteligente: desarrollo de una economía basada en el conocimiento y la innovación; (ii) crecimiento sostenible: promoción de una economía que haga un uso más eficaz de los recursos, que sea más verde y competitiva; (iii) crecimiento integrador: fomento de una economía con alto nivel de empleo que tenga cohesión social y territorial.

\footnotetext{
${ }^{33}$ La Declaración de Bethesda es el resultado de las conclusiones de una amplia representación de los agentes implicados en la investigación biomédica (científicos, sociedades científicas, bibliotecas, editores, instituciones y agencias financiadoras) sobre cómo proceder, de forma inmediata, ante el objetivo de dotar a las publicaciones científicas de acceso abierto. Esta reunión tuvo lugar en la sede del Howard Hughes Medical Institute en Chevy Chase, Maryland (EEUU), el 11 de abril de 2003.

${ }^{34}$ Se publicaba en el mundo millón y medio de artículos científicos y Europa estaba a la cabeza con un 41,3 del total mundial de las publicaciones científicas, frente al 31,4\% de EE.UU., pero, sin embargo, Europa estaba a la cola en cuanto al número de referencias para la mayor parte de las disciplinas. (IP/04/747: 1). ${ }^{35}$ El estudio fue encargado por la Dirección General de Investigación de la Comisión Europea a cuatro grupos de de trabajo de dos universidades, la Universidad Libre de Bruselas y la Universidad de Ciencias Sociales de Toulouse.

${ }^{36}$ De entre los que destacamos Report LEG, 2007.

${ }^{37}$ Objetivos señalados ya en el documento CCE 2005a y recogidos en el Libro Verde.
} 
De las líneas de acción sobre las que la Unión Europea abordará los puntos básicos destacados por la Comunidad nos interesa detenernos en el primero, el crecimiento inteligente, que conlleva el desarrollo de una economía basada en el conocimiento y la innovación. Esto significa la consolidación del conocimiento y la innovación como impulsores del crecimiento futuro, lo que requiere (i) mejorar la calidad de nuestra educación, (ii) consolidar los resultados de la investigación, (iii) promover la innovación y la transferencia de conocimientos en toda la Unión, (iv) explotar al máximo las TIC y asegurarse de que las ideas innovadoras puedan convertirse en nuevos productos y servicios que generen crecimiento y empleos de calidad. La realización de ese ambicioso objetivo va a exigir los países de la Unión un incremento de sus inversiones en $\mathrm{I}+\mathrm{D}$, en tecnologías (TIC) y una atención especial a la educación, formación y aprendizaje a lo largo de la vida. Para la década 2010-2020, la Unión Europea se ha propuesto llevar a cabo tres 'iniciativas emblemáticas': (i) unión por la innovación, (ii) juventud en movimiento y (iii) una agenda digital para Europa. El contenido que se ha dado a estas iniciativas es muy amplio, pero las metas están claras.

\section{Conclusiones}

Como hemos visto, el desarrollo de la acción de la Comunidad Europea sobre las revistas científicas se desarrolla, fundamentalmente, en los años de principal desarrollo del EEI y del EEES, aunque no dejan de producirse aportaciones a todas las iniciativas europeas propuestas. En la consulta sobre la futura estrategia UE 2020. (2009) se analizan los problemas que conlleva, en todos los ámbitos, la actual crisis. En relación con el Espacio Europeo en educación e investigación, el principio propuesto de reflexión es crear valor basando el crecimiento en el conocimiento, y en él se reconoce que el conocimiento es el motor del crecimiento sostenible. En un mundo en cambiante y competitivo, los factores que marcan la diferencia son la educación y la investigación, la innovación y la creatividad, es decir, la reflexión se vuelve a centrar en el triángulo del conocimiento. La UE debe fijar condiciones más atractivas para la innovación y la creatividad, especialmente previendo medidas que incentiven el crecimiento de las empresas basadas en el conocimiento ${ }^{38}$.

Si hemos de sintetizar cuáles son las necesidades de cambio de nuestras revistas de cara al futuro, posiblemente basten tres palabras para ello: renovación, reorganización e innovación, porque es necesario (i) renovar las revistas científicas en forma, contenido y difusión siguiendo parámetros de calidad y excelencia; (ii) reorganizar el actual conglomerado de revistas científicas en niveles, ramas de conocimiento y áreas temáticas en el contexto de la institución de acogida y del panorama institucional español; y (iii) innovar, adoptando los criterios marcados por la UE para converger y lograr una identidad en publicaciones científicas. La cuarta fase que se inicia ahora ha marcado ya las pautas que se van a seguir en educación y política científica en la próxima década. Todas las transformaciones que se han propuesto seguirán afectando, en cierta medida, a las publicaciones científicas que tendrán que continuar su adaptación para tener valor en la Europa del conocimiento a la que se tiende.

\section{Bibliografía}

[Proceso de Liubliana (2008). Consejo de Competitividad (2008). Hacia la plena realización del Espacio Europeo de Investigación. Doc. 10231/o8. Fecha de consulta, 30.05.2008.

Alonso, J., Subirats, I. y Martínez Conde, M.L. (2008). Informe APEI sobre acceso abierto. Fecha de consulta: 12.07.2010: http://eprints.rclis.org/15107/1/informeapeiaccesoabierto.pdf.

Bollen, Johan; Van de Sompel, Herbert; Hagberg, Aric.; Chute, Ryan. (2009). A principal component analysis of 39 scientific impact measures. PLoS ONE 4(6): e6o22. doi:10.1371/journal.pone.0006o22.http://arxiv.org/PS_c ache/arxiv/pdf/0902/0902.2183v1.pdf. Fecha de consulta, 26.02.2010.

British Academy (2009). Language Matters. A position paper. Fecha de consulta: 2.11.2009

http://www.britac.ac.uk/templates/assetrelay.cfm?frmAssetFileID $=8055$.

Carta ECHO. Charter ECHO (European Cultural Heritage Online), Berlín, el 30 de octubre de 2002. Fecha de consulta: 2.11.2009. http://echo.mpiwgberlin.mpg.de/policy/oa_basics/charter.

CCE (2000a). Hacia un espacio europeo de investigación. COM (2000) 6 final de 18.1.2000.

CCE (200ob). Realización del "Espacio europeo de la investigación": orientaciones para las acciones de la Unión en el ámbito de la investigación (2002-2006). COM (2000) 612 final de 4.10.2000.

CCE (2002). El Espacio Europeo de la Investigación: un nuevo impulso. COM (2002) 565 de 16.10.2002.

CCE (2005a). Acciones comunes para el crecimiento y el empleo: el programa comunitario sobre la estrategia de Lisboa. COM (2005) 330 final de 20.7.2005.

CCE (2005b). Una nueva estrategia marco para el multilingüismo. COM (2005) 596 final de 22.11.2005.

CCE (2007a). Sobre la información científica en la era digital: acceso, difusión y preservación. COM (2007) 56 final de 14.2.2007.

CCE (2007b). Libro Verde. El Espacio Europeo de Investigación: nuevas perspectivas.

CCE (2007c). Commission staff working document. Accompanying the Green Paper "The European Research Area: New Perspectives". Doc. 8322/07 [COM (2007) 161. Bruselas, 4.4.2007.

CCE (2008). Un Plan Europeo de Recuperación Económica. COM (2008) 800 final. de 26.11.2008.

CCE (2009). Documento de trabajo de la Comisión. Consulta sobre la futura estrategia "UE 2020”. COM (2009) 647 final de 24.11.2009.

CCE (2010). EUROPA 2020 Una estrategia para un crecimiento inteligente, sostenible e integrador. COM (2010) 2020 de 3.3.2010.

Comisión Europea (2006). DO L 236/28 de 31.8.2006 Sobre la digitalización y la accesibilidad en línea del material cultural y la conservación digital. Recomendación de la Comisión de 24 de agosto de 2006.

Conferencia de Berlín (2003). Realizando el Espacio Europeo de Educación Superior. Comunicado oficial de la Confe-

\footnotetext{
${ }^{38}$ Los informes encargados por la Comisión en los dos últimos años indican esas direcciones de actuación STC key figures report 2008/2009 y los documentos, ya citados, Kroll y Stahlecker 2009; Kroll, Zenker y Schubert 2009; Peter y Frietsch 2009.
} 
rencia de Ministros responsables en Educación Superior. Berlín, 19 de septiembre de 2003.

Conferencia de Budapest-Viena (2010). Budapest-Vienna Declaration on the European Higher Education Area. Viena, 12 de marzo de 2010.

Cossette, P. (2009). Publier dans une revue savante. Les 10 règles du chercheur convaincant. Québec, Presses de l' Université du Québec.

Declaración de Berlín (2003). Berlin Declaration on Open Access to Knowledge in the Sciences and Humanities. Berlin 22 de octubre de 2003. En Conference on Open Access to Knowledge in the Sciences and Humanities, 20 - 22 Oct 2003, Berlin, Sociedad Max Planck. Fecha de consulta: 2.11.200 http://oa.mpg.de/openaccessberlin/berlindeclaration.html.

Declaración de Bethesda 2003. Bethesda Statement on Open Access Publishing. Meeting on Open Access Publishing. Maryland, 11.04.2003. Fecha de consulta: 2.11.2009 http://www.earlham.edu/ peters/fos/bethesda.htm.

Declaración de Bolonia (1999). El Espacio Europeo de la Enseñanza Superior. Declaración conjunta de los ministros europeos de educación reunidos en Bolonia el 19 de junio de 1999.

Délégation générale à la langue française et aux langues de France (2009). Rapport au Parlement sur l'emploi de la langue française. Ministère de la Culture et de la Communication, París.

Delgado López-Cózar, E., Ruiz-Pérez, R. y Jiménez-Contreras, E. (2006). La Edición de Revistas Científicas Directrices, Criterios y Modelos de Evaluación. Madrid, FECYT.

Estrategia de Lisboa (2000). Consejo europeo de Lisboa 23 y 24 de marzo de 200o. Conclusiones de la Presidencia. DO C 205 de 17.7.2000.

Estrategia de Lisboa renovada (2005). Conclusiones de la Presidencia del Consejo Europeo de Bruselas (22 y 23 de marzo de 2005). Doc 7619/1/05 REV 1.

EURAB (2006). Scientific publication: policy on open access. EURAB 06.049, diciembre,2006.

Eurobarómetro 64.3 2006. Europeans and their Languages. Special Eurobarometer 243 / Wave 64.3 - TNS Opinion \& Social. Resumen en español EUROBARÓMETRO especial 243 "Los europeos y sus lenguas".

European Commission (2006). Study on the economic and technical evolution of the scientific publication markets in Europe (2006). European Commission, Directorate General for Research. http://ec.europa.eu/research/science-society/pdf/scientific-publication-study_en.pdf.

Fanelli Daniele (2010). Do Pressures to Publish Increase Scientists' Bias? An Empirical Support from US States Data. PLoS ONE 5(4): e10271. doi:10.1371/ journal.pone.0010271.

Fundación Española para la Ciencia y la Tecnología (2007). Criterios de calidad en la investigación en Humanidades. Informe 2007. FECYT.

Guillamón, A. (2006). Prólogo. En E., Ruiz-Pérez, R., Jiménez, E. (2007) Edición de Revistas Científicas: Directrices, Criterios y Modelos de Evaluación. Madrid: FECYT.
Heijs, J. (2009). ERAWATCH Country Report 2009. Analysis of policy mixes to foster $R \& D$ investment and to contribute to the ERA. Spain. Comision Europea JRC-IPTS, Luxemburgo.

IInformal Meeting (2008). Presidencia de la Unión Europea (2008). Informal Meeting of Ministers for Competitiveness (Research). Brdo, 15 April 2008. Draft summary by the Presidency. Fecha de consulta 2.11.2009. http://www.eu2008.si/en/News_and_Documents/down load_docs/April/0414_COMPET/o70Summary_Research.pdf.

Iniciativa de Budapest (2001). Budapest Open Access Initiative, BOAI. http://www.soros.org/openaccess.

IP/04/747. An effective scientific publishing system for European research. Bruselas, 15.06.2004.

Kroll, H., y Stahlecker, T. (2009). Europe's regional research systems: current trends and structures. Comunidad Europea, Luxemburgo.

Kroll, H., Zenker, A. y Schubert, T. (2009). An analysis of the development of $R \& D$ expenditure at regional level in the light of the 3\% target. Comunidad Europea, Luxemburgo.

LB Humanidades. Fundación Española para la Ciencia y la Tecnología (2006). Libro blanco de la investigación en Humanidades. FECYT.

Levitt, R., Janta, B., Shehabi, A., Jones, D., Valentini, E. (2009). Language matters. The supply of and demand for UK born and educated academic researchers with skills in languages other than English. Cambridge, RAND Europe. Libro Verde. V. CCE 2007b.

Pérez Sedeño, E. (2006).Presentación. En E. Delgado LópezCózar, R. Ruiz-Pérez y E. Jiménez-Contreras, E. (2006).

Peter, V. y Frietsch, R. (2009). Exploring regional structural and S\&T specialisation: implications for policy. Comunidad Europea, Luxemburgo.

Plan Nacional 2008-2011 (2007). Comisión Interministerial de Ciencia y Tecnología (2007). Plan Nacional de Investigación científica, Desarrollo e Innovación Tecnológica 2008-2001. CICYT.

Quinta libertad (2008). Presidencia de la Unión Europea (2008). Consejo Europeo de Bruselas, 13 y 14 de marzo de 2008. Conclusiones de la Presidencia. Doc 7652/1/08. REV 1.

Report LEG, (2007) Open research and innovation policies for Europe - A leap forward!. A Report of the Lisbon Expert Group. Comisión Europea, 4.04.2007.

Roldán López, A. (2009). Biliometría: JCR [blog de internet]. Madrid, Álvaro Roldán López. 18 junio 2010. Fecha de consulta 1 julio 2010. http://www.bibliometria.com/jcr-2009.

STC key figures report 2008/2009 (2008). Comisión Europea (2008). A more research-intensive and integrated European Research Area Science, Technology and Competitiveness key figures report 2008/2009. Comunidad Europea, Luxemburgo.

Visión 2020.Consejo de Competitividad (2008). Visión 2020 para el Espacio Europeo de Investigación. Conclusiones del Consejo de Competitividad. Doc 16767/o8. RECH 410. COMPET 550. Bruselas 2.12.2008. 